\title{
La importancia de los metabolitos secundarios en el control de nematodos gastrointestinales en ovinos
} con énfasis en Yucatán, México

\section{The importance of secondary metabolites in the control of gastrointestinal nematodes in sheep with emphasis on Yucatán, Mexico}

\author{
Sepúlveda-Vázquez Jerónimoํㅜ, Torres-Acosta Juan Felipe ${ }^{2}$, Sandoval-Castro Carlos Alfredo²,

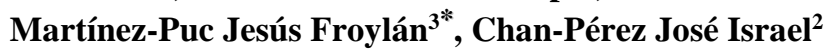

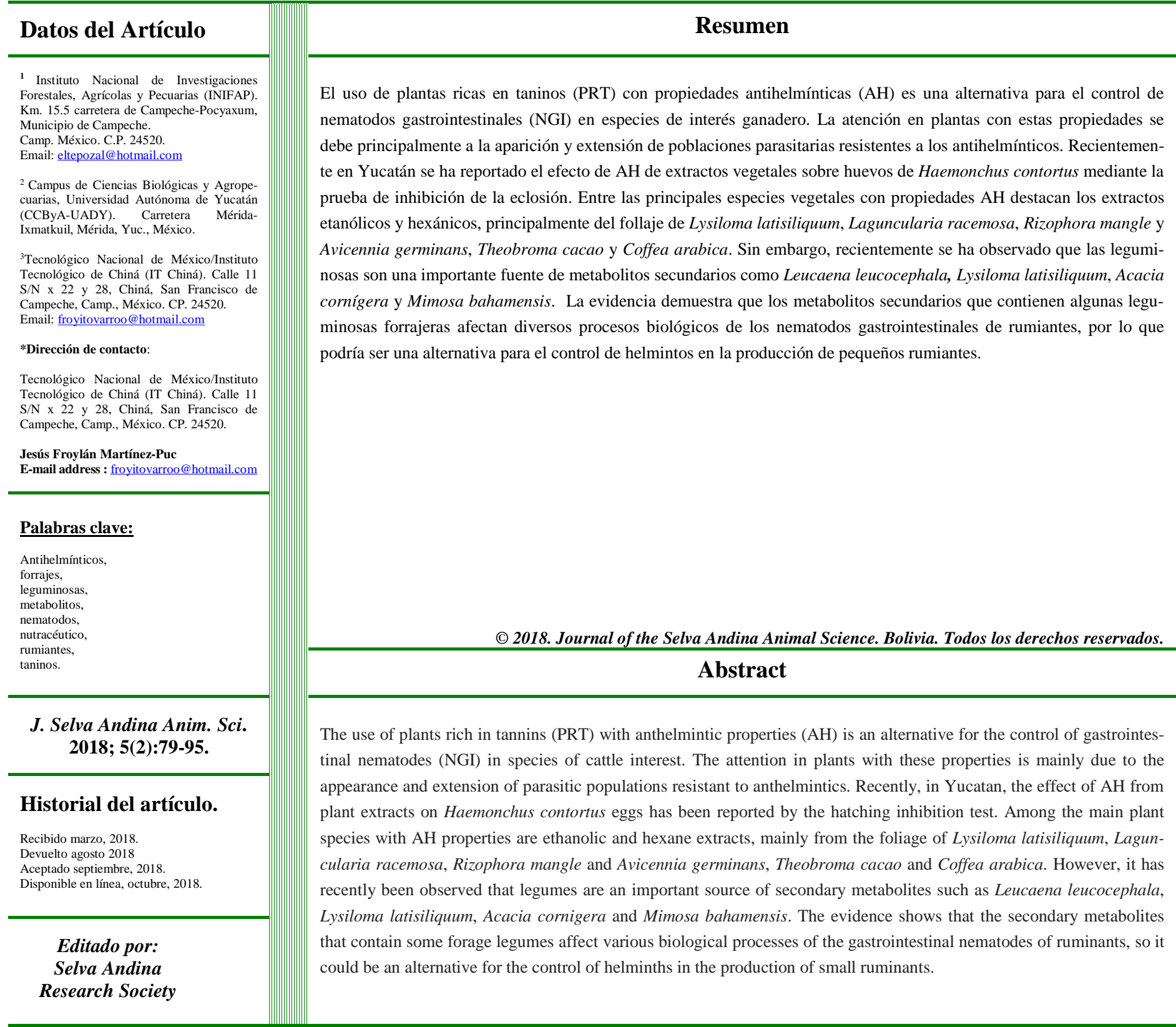




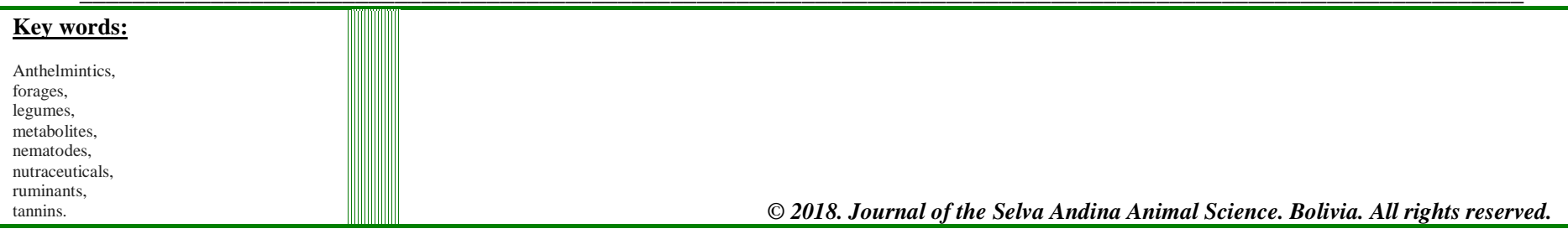

\section{Introducción}

Una limitante de importancia económica para el desarrollo de la ganadería bajo las condiciones del trópico son las infestaciones por parásitos gastrointestinales $^{1}$, que se reflejan en baja conversión alimenticia, mortalidad de los animales, reducción de los parámetros productivos y reproductivos, además del alto costo para su control, entre otros, que deriva en pérdidas económicas para los productores. ${ }^{2}$

Por mucho tiempo, la principal estrategia empleada por los productores para el control de parásitos gastrointestinales en rumiantes fue la aplicación de compuestos antihelmínticos sintéticos. Estos fármacos por su gran eficacia, amplio espectro y poder residual, tuvieron un éxito inicial, e incluso se llegó a pensar en que estos serían la solución final para la presencia de NGI, creando un falso sentido de seguridad en los productores, quienes sustituyeron el diagnóstico y el asesoramiento profesional, por la casi exclusiva utilización de drogas en forma irracional e indiscriminada. ${ }^{1}$

La aparición y extensión de poblaciones parasitarias en diversas especies resistentes a los antihelmínticos, la creciente preocupación por la inocuidad alimentaria, así como el impacto negativo sobre el medio ambiente de los fármacos antihelmínticos, son las principales razones que impulsaron la búsqueda de métodos alternativos al uso de fármacos sintéticos en el control de NGI en pequeños rumiantes. ${ }^{3-4}$ Por tal motivo, el uso de plantas ricas en taninos (PRT) con propiedades antihelmínticas (AH) es una opción para el control de NGI en especies de interés ganadero ${ }^{5-7,3,8,9}$, por lo que estas podrían desempeñar un papel preponderante en el control no convencional de las parasitosis en el futuro.

Los compuestos bioactivos (CBA) de las plantas tienen la capacidad de interactuar con proteínas de NGI, modificando sus propiedades físicas y químicas. ${ }^{3}$ Existen diversos métodos que han sido utilizados in vitro e in vivo, para validar y comprender sus propiedades AH de las plantas. ${ }^{10}$ Recientemente en Yucatán, se reportó el efecto AH de extractos vegetales sobre huevos de $H$. contortus mediante la prueba de inhibición de eclosión ${ }^{11}$, evaluando extractos etanólico, hexánico y diclorometano de Phytolacca icosandra, reportándose diferencias del efecto AH de cada extracto, teniendo una mayor eficacia los extractos etanólicos y hexánicos. Por otra parte ${ }^{12}$, evaluaron extractos hidro-acetónicos del follaje de Lysiloma latisiliquum, Laguncularia racemosa, Rizophora mangle y Avicennia germinans. Así mismo, semilla y cascarilla de Theobroma cacao y Coffea arabica, resultando mayor efecto AH en los extractos de $R$. mangle y cáscara de $T$. cacao.

Existe una amplia variedad de CBA. Sin embargo, se ha considerado a los taninos como principal agente responsable de la acción AH de las plantas, aunque se han reportado diversos compuestos secundarios principalmente saponinas, glucósidos ${ }^{10}, \mathrm{y}$ lectinas que presentan actividad AH sobre NGI de rumiantes. No obstante, se ha reportado una enorme variabilidad en la susceptibilidad de los NGI hacia 
diversas plantas y sus derivados. ${ }^{13}$ Esta variación en la respuesta $\mathrm{AH}$ ha sido estudiada por diversos autores, quienes atribuyen las variaciones del efecto $\mathrm{AH}$ a la especie y procedencia del $\mathrm{NGI}^{14,15,12}$, al tipo de hospedero ${ }^{16}$ así como a factores propios de las plantas y del tipo de bioensayo. ${ }^{17}$

En recientes estudios realizados en el estado de Yucatán, fue posible determinar que $H$. contortus, aislados en la región, presentan menor susceptibilidad in vitro a extractos acetónicos ricos en taninos obtenidos de plantas de la vegetación local, en comparación con otros aislados de la misma especie provenientes de regiones templadas que los pequeños rumiantes no han sido expuestos a este tipo de vegetación. ${ }^{15,12}$ Estos resultados sugieren que la exposición previa de los NGI a los metabolitos secundarios de las plantas ha propiciado la generación de resistencia en los parásitos, ocasionando variaciones en la susceptibilidad en diferentes aislados. Así mismo ${ }^{18}$, registraron las especies de plantas forrajeras presentes en la vegetación nativa que son consumidas por los pequeños rumiantes en pastoreo, así como la forma y tamaño de bocados, logrando determinar el nivel de consumo. Se presume que la susceptibilidad de los NGI hacia los extractos de plantas taníferas pudiera estar influenciado por el historial y nivel de su consumo, por parte de los rumiantes, presentándose el mayor efecto $\mathrm{AH}$ hacia las que se tiene menor consumo y/o historial de exposición. A pesar que se ha propuesto que la exposición previa a las plantas influye sobre la susceptibilidad de $H$. contortus, esta hipótesis no ha sido probada ampliamente, ni se ha establecido si existe una relación entre el nivel de consumo de las plantas y el grado de susceptibilidad in vitro de $H$. contortus hacia las mismas.

\section{Metabolitos secundarios (MS)}

Las plantas, a diferencia de otros organismos, destinan una cantidad significativa del carbono asimilado y de la energía a la síntesis de una amplia variedad de moléculas orgánicas que no parecen tener una función directa en los procesos metabólicos primarios como la fotosíntesis, respiración, asimilación de nutrientes, transporte de solutos o síntesis de proteínas, carbohidratos o lípidos, y las cuales se denominan como metabolitos secundarios. ${ }^{19}$

Se considera que existen alrededor de 1200 compuestos secundarios, presentando una distribución variable dentro del reino vegetal. Generalmente cada tipo de MS se presenta en una sola especie o grupo de plantas taxonómicamente relacionadas..$^{20-22}$ Los MS tienen diversas funciones, que están relacionadas con las interacciones ecológicas entre planta y medio ambiente, principalmente en defensa de la planta contra depredadores y patógenos, actuando como atrayentes o repelentes de animales, propiciándole sabor amargo a las plantas, reducir su digestibilidad y palatabilidad en los herbívoros e incluso convirtiéndolas en tóxicas para estos, inhibiendo así el desarrollo de insectos, nematodos, hongos, bacterias, mejorando el crecimiento de las plantas y la consistencia. ${ }^{23,20,19,24}$ También protegen a las plantas del estrés abiótico, como las radiaciones ultravioletas. ${ }^{25}$

En menor medida, los MS también actúan dentro de la reproducción como atrayentes de polinizadores y animales que se alimentan de los frutos, como pigmentos de flores y/o frutos ${ }^{19}$, así como también participan en la comunicación de las plantas con otros organismos. ${ }^{25}$

La mezcla de metabolitos secundarios es única para cada especie ${ }^{19}$, presentando variaciones a lo largo del año ${ }^{22}$, ya que diversos factores, como radiación 
solar, edad, estado fenológico de la planta, nutrición, estrés hídrico, procedencia geográfica, precipitación, interacción con herbívoros, condiciones de recolección del vegetal interacciones bióticas entre otras, influyen para que genere mecanismos de adaptación entre ellos, que determinan la producción de $\mathrm{MS}{ }^{23} \mathrm{Se}$ sabe que estos compuestos son más comunes en la vegetación tropical que en los ecosistemas templados, y los ambientes húmedos en comparación a los secos. ${ }^{26}$ Por otra parte, además de la variación estacional, los MS no se distribuyen uniformemente entre todos sus componentes estructurales, al igual, en las yemas en crecimiento, hojas jóvenes, órganos reproductores, dispersión, y en general todas las partes en crecimiento anual, existe mayor concentración, reactividad y diversidad de MS. ${ }^{26}$ Las plantas rastreras contienen menores concentraciones de metabolitos secundarios que las arbóreas, tabla 1..$^{22,27}$ (Tabla 1)

Tabla 1 Distribución de metabolitos secundarios en diversas especies vegetales

\begin{tabular}{|c|c|c|c|c|c|c|c|c|c|c|c|c|c|c|c|}
\hline Planta & Tipo de extracto & Es & $\mathbf{T p}$ & $\mathbf{C m}$ & Sp & Al & Tn & $\mathbf{T r}$ & $\mathbf{C l}$ & Fn & An & $\mathbf{C a}$ & $\mathbf{F t}$ & Pr & Referencia \\
\hline \multirow{3}{*}{ Phytolacea icosandra. } & Etanólico & $\mathrm{X}$ & $\mathrm{X}$ & $\mathrm{X}$ & $\mathrm{X}$ & & & & & & & & & & \multirow{3}{*}{ Hernández-Villegas et al. ${ }^{11}$} \\
\hline & Diclorometano & $\mathrm{X}$ & $\mathrm{X}$ & $\mathrm{X}$ & & & & & & & & & & & \\
\hline & Hexánico & $\mathrm{X}$ & $\mathrm{X}$ & $\mathrm{X}$ & & & & & & & & & & & \\
\hline Annona squamosa. & Metanólico & & & & & & $X$ & & & & & & & & \multirow{4}{*}{ Kamaraj \& Rahuman. ${ }^{56}$} \\
\hline Eclipta prostrata. & Metanólico & & & & & & $\mathrm{X}$ & & & & & & & & \\
\hline Terminalia chebula. & Acetónico & & & & & & & $\mathrm{X}$ & & & & & & & \\
\hline Catharanthus roseus. & Acetónico & $\mathrm{X}$ & & & & $\mathrm{X}$ & & & & & & & & & \\
\hline Manihot esculenta. & $\begin{array}{c}\text { Acuoso, metanólico y } \\
\text { diclorometano }\end{array}$ & & & $\mathrm{X}$ & & & $\mathrm{X}$ & & & & & & & $\mathrm{X}$ & Marie-Magdeleine et al..$^{57}$ \\
\hline \multirow[t]{2}{*}{ Mimosa caesalpiniifolia. } & Etanólico & & $\mathrm{X}$ & & & & $\mathrm{X}$ & & & $\mathrm{X}$ & & $\mathrm{X}$ & & & Silva et al..$^{58}$ \\
\hline & Hexánico & & & & & & & & & & & & $\mathrm{X}$ & & \multirow{3}{*}{ Monteiro et al. ${ }^{59}$} \\
\hline \multirow[t]{2}{*}{ Jatropha curcas. } & Acetónico & & & & & & & & & & & & $X$ & & \\
\hline & Etanólico & & & & & & $\mathrm{X}$ & $\mathrm{X}$ & & & & $\mathrm{X}$ & $\mathrm{X}$ & & \\
\hline \multirow[t]{2}{*}{ L. leucocephala. } & $\begin{array}{l}\text { Metanólico, hexánico } \\
\text { y acetónico }\end{array}$ & $\mathrm{X}$ & & & & $\mathrm{X}$ & & $\mathrm{X}$ & $\mathrm{X}$ & & & & & & \multirow{2}{*}{$\begin{array}{l}\text { Chen \& Wang. }{ }^{60} \\
\text { Chew et al. }{ }^{61}\end{array}$} \\
\hline & Metanólico & & & & & $\mathrm{X}$ & $\mathrm{X}$ & & & & & & & & \\
\hline Acacia albida. & \multirow{3}{*}{ Acuoso } & & & & $\mathrm{X}$ & $\mathrm{X}$ & & & & & & & & & \multirow{3}{*}{ Alhaji et al. ${ }^{62}$} \\
\hline Acacia nilotica. & & & & & $\mathrm{X}$ & & $\mathrm{X}$ & & & & & & & & \\
\hline Acacia seyal. & & & & & $\mathrm{X}$ & & $\mathrm{X}$ & & & $\mathrm{X}$ & $\mathrm{X}$ & & & & \\
\hline
\end{tabular}

\section{Consumo de metabolitos secundarios por los rumiantes}

Actualmente, los sistemas de producción ganadera consideran que la nutrición apropiada se puede proporcionar a través del monocultivo. Sin embargo, los rumiantes pueden satisfacer sus necesidades de nutrientes y regular su ingesta de compuestos secundarios cuando se les ofrece una variedad de alimentos. Esta variedad es fundamental, ya que las plantas les proporcionan una gran variedad de productos químicos, con un potencial de mejorar su salud y el bienestar. Por ejemplo, los MS, que protegen a las plantas de depredadores y plagas, también afectan negativamente los procesos celulares y metabólicos de los herbívoros. Empero, a dosis bajas en mezclas apropiadas, estos compuestos pueden presentar efectos beneficiosos sobre la salud y nutrición animal. 
La variedad de alimentos permite que los animales expresen sus preferencias alimentarias, que a la vez mejoran su bienestar, mediciones conductuales pueden representar una herramienta importante para evaluar este efecto en el ganado. ${ }^{28}$ En el estado de Yucatán ${ }^{18}$, registraron especies, así como la forma y tamaño de plantas forrajeras consumidas por pequeños rumiantes pastoreando para determinar el nivel de consumo. Las observaciones realizadas durante tres días consecutivos en dos ocasiones al mes, con intervalo de al menos diez días entre cada observación durante un año. Se acompañó a un rebaño mixto compuesto por ovejas y cabras, en los circuitos de pastoreo cotidiano por cuatro horas al día. El consumo de todos los materiales vegetales se registró en tres ovejas y tres cabras previamente habituadas a la presencia de los observadores humanos. Los datos se registraron en diferentes categorías de acuerdo al grupo taxonómico y el tamaño de cada bocado.

\section{Efecto antihelmíntico de los metabolitos secun- darios}

Debido al importante impacto económico de los NGI en la industria ovina mundial ${ }^{4}$ y a raíz de la aparición de la resistencia antihelmíntica (RAH), ha surgido el interés por estudiar las propiedades antihelmínticas de diversos MS de las plantas, entre ellos los taninos, como una alternativa para el control de NGI de rumiantes. ${ }^{29,30} \mathrm{El}$ efecto ocasionado por el consumo de plantas taníferas sobre las poblaciones de NGI pueden clasificarse en directos e indirectos. ${ }^{31}$ Los efectos directos son las interacciones que tienen sobre las funciones fisiológicas de los NGI, interfiriendo sobre los procesos de eclosión, desarrollo y motilidad de larvas infectantes, alimentación y reproducción de las fases adul83 tas. ${ }^{17,432,12}$ Por otra parte, los efectos indirectos constituyen la mejora en la absorción de proteínas, ya que los MS favorecen que estas sean asimiladas a nivel de duodeno. Se ha demostrado que una mayor asimilación de proteínas está asociada con la mejora en la inmunidad del hospedero. ${ }^{31}$ Existe variabilidad en los valores de respuesta que presentan los extractos de las plantas ricas en taninos (PRT) sobre NGI, asociados principalmente con la dinámica temporal y espacial que se observa en la concentración y composición de los compuestos polifenólicos en la naturaleza. ${ }^{4}$

Otra fuente de variación la constituye la modalidad del bioensayo que se utiliza para validar la acción AH de los compuestos secundarios. Generalmente los estudios se han llevado a cabo in vitro, bajo la suposición de que la intensidad y el tipo de efecto observado en contra los parásitos sea similar al que se presentará en el hospedero. Este tipo de pruebas presentan varias ventajas, como la obtención de resultados a menor tiempo e inversión, con mayor control de las condiciones, son fáciles de realizar y repetir, además, se pueden realizar en diversas etapas del ciclo biológico de los nematodos, pueden ser evaluados extractos enteros o purificados sin el riesgo de inferencia en los resultados a causa de otros componentes de las plantas (Figura 1). Sin embargo, en la mayoría de los casos, el material debe ser extraído de la planta, por lo que de acuerdo al solvente utilizado, será el tipo y cantidad de MS en el extracto, además en ocasiones, la modalidad in vitro no es posible reproducir totalmente las condiciones ambientales que se presentarán in vivo, por la diferencia fisiológicas entre el sitio de predilección del parásito en el hospedero, así como factores propios del hospedero que afectan la biodisponibilidad de los compuestos bioactivos. 
Figura 1 Factores que influyen en la variabilidad del efecto $\mathrm{AH}$ de los compuestos bioactivos presentes en las plantas

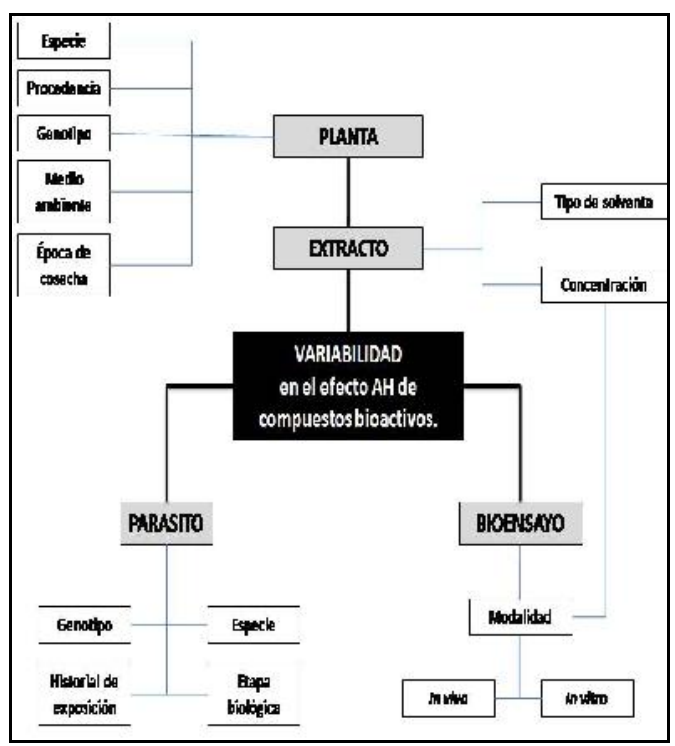

Por lo anterior se consideran más confiables los resultados obtenidos de estudios in vivo que los in vitro. Sin embargo, los ensayos in vitro son de gran utilidad, ya que representan un primer acercamiento en la selección inicial de especies y concentraciones más adecuadas para llevar a cabo ensayos en condiciones in vivo. ${ }^{10}$

Un factor a considerar en la variación del efecto de los MS, tiene origen en la metodología que se emplea para su evaluación. La técnica de extracción de los MS es uno de los principales elementos a considerar, ya que esta juega un rol importante en su evaluación, debido a que la metodología y solvente utilizados en la extracción influyen sobre el tipo y cantidad de metabolitos presentes en el extracto. ${ }^{33}$ (Tabla 2)

Tabla 2 Tipos de metabolitos secundarios extraídos empleando distintos solventes

\begin{tabular}{|c|c|c|c|c|c|c|}
\hline \multicolumn{7}{|c|}{ Tipo de solvente } \\
\hline Agua & Metanol & Acetona & Etanol & Cloroformo & Diclorometano & Eter \\
\hline $\begin{array}{c}\text { Antocianinas. } \\
\text { Taninos. } \\
\text { Saponinas. } \\
\text { Terpenoides. }\end{array}$ & $\begin{array}{c}\text { Antocianinas. } \\
\text { Terpenoides. } \\
\text { Saponinas. } \\
\text { Taninos. } \\
\text { Flavonoides. } \\
\text { Polifenoles. }\end{array}$ & Flavonoides. & $\begin{array}{l}\text { Taninos. } \\
\text { Polifenoles. } \\
\text { Flavonoides. } \\
\text { Terpenoides. } \\
\text { Alcaloides. }\end{array}$ & $\begin{array}{l}\text { Terpenoides. } \\
\text { Flavonoides. }\end{array}$ & Terpenoides. & $\begin{array}{l}\text { Terpenoides. } \\
\text { Alcaloides. }\end{array}$ \\
\hline
\end{tabular}

Azmir et al. ${ }^{33}$

efecto AH sobre la migración larvaria de cuatro

La actividad nematicida de los extractos ricos en taninos de diversas leguminosas y plantas leñosas ha sido probada en modalidad in vitro. ${ }^{34}$ Incluso se ha descrito el efecto $\mathrm{AH}$ in vivo que resulta de consumir los forrajes de las plantas ricas en taninos. ${ }^{35-37}$ Varios estudios in vitro han señalado la influencia que tienen los taninos condensados sobre los NGI de los ovinos al afectar diversos procesos biológicos de éstos. ${ }^{15,35,12}$ Así mismo, también se han compara

do la susceptibilidad de aislados de $H$. contortus de diferente procedencia geográfica ${ }^{15}$, evaluaron el plantas ricas en taninos (PRT) locales (Acacia pennatula, L. latisiliquum, Piscidia piscipula y L. leucocephala), los resultados permitieron sugerir la adaptación del aislado Campus de Ciencias Biológicas y Agropecuarias- Universidad Autónoma de Yucatán (CCByA-UADY )hacia los extractos de plantas ricas en taninos (PRT) de Yucatán, al encontrar que este era menos sensible que los aislados Centro Nacional de Investigación DisciplinariaInstituto Nacional de Investigaciones Forestales, Agrícolas y Pecuarias y Facultad de Estudios Superiores de Cuautitlán-Universidad Nacional Autóno- 
ma de México CENID-INIFAP y FESC-UNAM. Resultados similares fueron reportados por ${ }^{12}$, quienes evaluaron las diferencias en la susceptibilidad de seis aislados de $H$. contortus procedentes de México y Francia hacia extractos de subproductos agroindustriales y de PRT. Los resultados obtenidos sugieren una menor susceptibilidad de aislados de $H$. contortus con historial de contacto con PRT. Los resultados de ambos estudios sugieren que NGI presentan una adaptación hacia las plantas forrajeras nativas, esto quizás por la exposición constante, lo que genera una presión en la selección, eliminando selectivamente a NGI susceptibles de la población genéticamente heterogénea, produciéndose un incremento de individuos portadores de genes que confieren resistencia a los metabolitos secundarios y son transmitidos a la siguiente generación. ${ }^{38}$ (Tabla 3).

Entre los bioensayos in vitro, se encuentra la inhibición de eclosión de huevos (Egg Hatch Assay, EHA por sus siglas en ingles). Originalmente, esta prueba fue diseñada para la evaluación del efecto $\mathrm{AH}$ de los benzimidazoles, pero en la actualidad, también se emplea para determinar el efecto $\mathrm{AH}$ de compuestos secundarios de las plantas. La prueba consiste en incubar por $48 \mathrm{~h}$, huevos de NGI en concentraciones seriadas de compuestos con propiedades $\mathrm{AH}$, para posteriormente contabilizar el porcentaje de huevos que eclosionan en cada una de las concentraciones, los resultados se contrastan con el grupo control, para determinar la eficacia se calcula la concentración eficaz $50\left(\mathrm{CE}_{50}\right)$, que es la concentración requerida para evitar la eclosión del 50\% de los huevos. ${ }^{39}$

Se han descrito dos efectos diferentes de los $\mathrm{AH}$ en contra de los huevos de nematodos: i) impidiendo la formación del embrión o ii) impidiendo la salida de la larva del huevo (inhibición de eclosión). Este 85 ensayo tiene la ventaja de que permite obtener resultados en forma rápida, ya que los huevos que se utilizan en ella, son obtenidos en el momento de un animal donador. Presenta la desventaja de la obtención de huevos al ser necesario procesar a las heces en un lapso no mayor a $3 \mathrm{~h}$, ya que de lo contrario se corre el riesgo de que las larvas se desarrollen y eclosionen. ${ }^{40,39}$ (Tabla 4).

\section{Plantas ricas en taninos del estado de Yucatán}

Las áreas de vegetación secundaria nativa son fuente de nutrientes para los pequeños rumiantes en pastoreo. ${ }^{41}$ En el estado de Yucatán la vegetación secundaria, abarca extensiones de plantaciones de henequén abandonadas, también áreas agrícolas de temporal que es practicada en las comunidades rurales. En Yucatán se considera que este tipo de ecosistema está compuesto por alrededor de 1980 especies florísticas, de ellas 201 son reportadas con potencial forrajero, siendo la mayoría leguminosas. ${ }^{42,43}$ Los animales que pastorean en vegetación secundaria deben utilizar una comunidad mucho más diversa y variable con respecto a las praderas de gramíneas. Esta variabilidad permite al herbívoro la posibilidad de seleccionar su dieta en base a sus necesidades. ${ }^{41}$ Recientemente en Yucatán, se realizan observaciones para determinar el nivel de consumo de pequeños rumiantes en pastoreo en vegetación heterogénea nativa ${ }^{44,18}$, a la fecha ha sido posible identificar las especies y el nivel de su consumo (Tabla 5). 


\section{Tabla 3 Reporte de efectos antihelmínticos de los taninos sobre NGI en investigaciones previas en México}

\begin{tabular}{|c|c|c|c|c|c|}
\hline Referencia & Especie & Helminto utilizado & Tipo de extracto & Técnica empleada & Resultados \\
\hline Calderón-Quintal et al. ${ }^{15}$ & $\begin{array}{l}\text { Acacia pennatula, L. latisiliquum, } \\
\text { Piscidia piscipula y } L . \text { leucocepha- } \\
\text { la }\end{array}$ & $\begin{array}{l}\text { H. contortus } \\
\text { CENID-INIFAP, UADY } \\
\text { y UNAM. }\end{array}$ & Hidro-acetónicos & $\begin{array}{l}\text { Prueba de inhibición de la } \\
\text { migración larvaria. }\end{array}$ & $\begin{array}{r}\text { Las larvas obtenidas del estado de Yuc., Méx., son menos sensibles a } \\
\text { los extractos de PRT obtenidos de la misma región sugiriendo una } \\
\text { posible adaptación de los parásitos a los taninos. }\end{array}$ \\
\hline Cobarruvias-Cardenas et al. $^{63}$. & A. pennulata y $C$. arabica. & $\begin{array}{l}\text { H. contortus } \\
\text { (FESC-UNAM). }\end{array}$ & Hidro-acetónicos & $\begin{array}{r}\text { Inhibición del desenvaine y de la } \\
\text { migración larvaria }\end{array}$ & $\begin{array}{r}\text { Se encontró una variación en el efecto antihelmíntico de los extractos } \\
\text { utilizados, teniendo mayor susceptibilidad hacia los extractos ricos en } \\
\text { taninos provenientes de especies vegetales tropicales en comparación } \\
\text { con los extractos de subproductos agroindustriales. }\end{array}$ \\
\hline Ortiz-Ocampo et al. ${ }^{64}$ & A. pennulata y $C$. arabica. & $\begin{array}{l}\text { H. contortus (Paraíso, } \\
\text { Yucatán). }\end{array}$ & Hidro-acetónicos & $\begin{array}{r}\text { Inhibición de la migración } \\
\text { larvaria y del desenvaine }\end{array}$ & $\begin{array}{r}\text { Se observó diferencias en el nivel de concentración en la cual se } \\
\text { observa el efecto AH para cada una de las pruebas. }\end{array}$ \\
\hline Vargas-Magaña et al. ${ }^{12}$ & $\begin{array}{l}\text { L. latisiliquum, L. racemosa, } R . \\
\text { mangle, Avicennia germinans, } T . \\
\text { cacao y } C \text {. arabica. }\end{array}$ & $\begin{array}{l}\text { H. contortus } \\
\text { (CENID-INIFAP). }\end{array}$ & Hidro-acetónicos & $\begin{array}{r}\text { Inhibición del desarrollo larvario } \\
\text { y de la eclosión. }\end{array}$ & $\begin{array}{l}\text { Los extractos ricos en taninos no inhiben significativamente el desarro- } \\
\text { llo de las larvas de NGI dentro del huevo, contrario a lo observado en la } \\
\text { eclosión donde se encontró un efecto antihelmíntico. }\end{array}$ \\
\hline Martínez-Ortiz-de-Montellano et al.$^{65}$ & $\begin{array}{l}\text { L. latisiliquum y Onobrychis } \\
\text { viciifolia }\end{array}$ & H. contortus & Hidro-acetónicos & $\begin{array}{l}\text { Bromatología, observación } \\
\text { directa, registro de consumo }\end{array}$ & $\begin{array}{r}\text { Se observó que los NGI expuestos a taninos presentaron modificacio- } \\
\text { nes estructurales en la cutícula y zona bucal, lo que podría afectar su } \\
\text { alimentación y motilidad. }\end{array}$ \\
\hline Galicia-Aguilar et $a l^{36}$ & H. albicans & $\begin{array}{l}\text { H. contortus } \\
\text { CENID-INIFAP }\end{array}$ & Follaje fresco & $\begin{array}{l}\text { Registro diario de datos, necrop- } \\
\text { sia y microscopia directa }\end{array}$ & $\begin{array}{l}\text { Se observó una reducción en la longitud hembras, así como en la } \\
\text { fecundidad (huevos en el útero). En ejemplares de H. contortus recupe- } \\
\text { rados después del sacrificio humanitario de los hospederos que consu- } \\
\text { mieron H. albicans. }\end{array}$ \\
\hline
\end{tabular}

\section{Tabla 4 Algunos estudios para detección de efecto AH de extractos de plantas hacia NGI mediante la técnica de Inhibición de la eclosión}

\begin{tabular}{|c|c|c|c|c|c|}
\hline Referencia & Origen/Planta & NGI & Aislado & Extracto & Resultados y Conclusiones \\
\hline Eguale et al. ${ }^{66}$ & $\begin{array}{r}\text { Coriandrum sativum } \\
\text { (Etiopia). }\end{array}$ & H. contortus & $\mathrm{NE}$ & Acuoso e hidroalcohólico & $\begin{array}{l}\text { Se emplearon dos tipos de extractos de semillas } C \text {. sativum. Ambos inhibieron completamente la eclosión a } \\
\text { concentración de } 0.5 \mathrm{mg} \text {. La DE } \mathrm{D} \text { a del extracto acuoso fue de } 0.12 \mathrm{mg} / \mathrm{ml} \text { y la del extracto hidro-alcohólico en } \\
0,18 \mathrm{mg} / \mathrm{ml} \text {. No hubo diferencias estadísticamente significativas entre los extractos }(\mathrm{p}>0,05) \text {. El extracto hidro- } \\
\text { alcohólico mostró mejor actividad in vitro contra parásitos adultos. En el experimento in vivo se mostró un mode- } \\
\text { rado efecto } \mathrm{AH} \text { en la reducción de huevos fecales y conteo de nematodos adultos, }\end{array}$ \\
\hline $\begin{array}{l}\text { Marie-Magdeleine } \\
\text { et al. }\end{array}$ & $\begin{array}{r}\text { Manihot esculenta } \\
\text { (Antillas Francesas). }\end{array}$ & H. contortus & $\mathrm{NE}$ & $\begin{array}{r}\text { Acuoso, diclorometano y } \\
\text { metanólico }\end{array}$ & $\begin{array}{l}\text { Se utilizaron tres tipos de extractos (acuoso, diclorometano y metanólico) en cuatro tipos de bioensayo. En I. de } \\
\text { eclosión, desarrollo, migración y de motilidad no se observaron efecto AH para ninguno de los tres tipos de } \\
\text { extractos. Solo en I. del desarrollo hubo efecto hacia el extracto metanólico. Los resultados sugieren que los } \\
\text { extractos tienen efecto sobre algunas fases de } H \text {. contortus y que los principios activos responsables de la activi- } \\
\text { dad podrían ser los terpenoides y taninos condensados. }\end{array}$ \\
\hline Eguale et al. ${ }^{66}$ & $\begin{array}{r}\text { Senna occidenta- } \\
\text { lis, Leonotis ocymifo- } \\
\text { lia, Leucas martinicen- } \\
\text { sis, Rumex abyssi- } \\
\text { nicus y Albizia schimpe- } \\
\text { riana. } \\
\text { (Etiopia). }\end{array}$ & H. contortus & $\mathrm{NE}$ & Acuoso e hidro-alcohólico & $\begin{array}{l}\text { Los extractos acuoso e hidro-alcohólico de Leucas martinicensis y Leonotiso cymifolia, así como el extracto } \\
\text { acuoso de Senna occidentalis y Albizia schimperiana generan una completa inhibición de la eclosión de los } \\
\text { huevos a una concentración de menos de o igual a } 1 \mathrm{mg} / \mathrm{ml} \text {. Los extractos acuosos e hidro-alcohólicos de todas } \\
\text { las plantas evaluadas han demostrado en forma estadística, una dependencia entre la dosis y la inhibición de la } \\
\text { eclosión. Con base en la DL } \mathrm{D} \text { so, los extractos más potentes fueron los de tipo acuoso } 2 \text { hidro-alcohólicos de Leucas } \\
\text { martinicensis }(0,09 \mathrm{mg} / \mathrm{ml}) \text {, así como los extractos acuosos de Rumex abyssinicus }(0,11 \mathrm{mg} / \mathrm{ml}) \text { y Albizia } \\
\text { schimperiana }(0,11 \mathrm{mg} / \mathrm{ml}) \text {. }\end{array}$ \\
\hline $\begin{array}{l}\text { Hernández- } \\
\text { Villegas } \\
\text { et al. }{ }^{11}\end{array}$ & $\begin{array}{r}\text { Phytolacca icosandra } \\
\text { (México). }\end{array}$ & H. contortus & CENID & $\begin{array}{r}\text { Etanólico, hexánico y diclo- } \\
\text { rometano }\end{array}$ & $\begin{array}{l}\text { Los extractos de tipo etanólico y diclorometano de } P \text {. icosandra mostraron un claro efecto } \mathrm{AH} \text { in vitro frente a } \\
\text { larvas } \mathrm{L}_{3} \text { y huevos de } H \text {. contortus. El extracto hexánico de } P \text {. icosandra no logró mostrar actividad AH in vitro. } \\
\text { La actividad AH de } P \text {. icosandra puede ser atribuida a las cumarinas, flavonoides, esteroides, terpenoides y / } \\
\text { saponinas o ben, a la interacción combinada de todos los componentes. El potencial de efectos tóxicos } \\
\text { de } P \text {. icosandra (propiedades hemolíticas) en los animales aún no se han investigado. }\end{array}$ \\
\hline
\end{tabular}


Tabla 5 Especies forrajeras con diferente nivel de consumo por cabras en pastoreo

\begin{tabular}{ccc}
\hline $\begin{array}{c}\text { Nivel de } \\
\text { consumo }\end{array}$ & Especie & $\begin{array}{c}\text { Nivel de } \\
\text { consumo (\%) }\end{array}$ \\
\hline Alto & L. leucocephala & 12.21 \\
(Mayor a 10\%) & G. floribundum & 11.24 \\
Medio & M. bahamensis & 3.24 \\
$(1-10 \%)$ & A. cornígera & 0.6 \\
Bajo & L. latisiliquum & 0.58 \\
(Menor a 1\%) & A. gaumeri & 0.13 \\
\hline & \multicolumn{2}{c}{ (González-Pech et al. datos por publicar) }
\end{tabular}

\section{Gymnopodium floribundum Rolfe}

Es una especie nativa de Mesoamérica, pertenece a la familia Polygonaceae Juss, conocida en la Península de Yucatán con el nombre maya de Dzidzilché. Un arbusto o árbol pequeño con alrededor de $3 \mathrm{~m}$ de alto, presenta una corteza profundamente fisurada, hojas alternas y simples, de ovadas a elípticas, peciolo corto, base redondeada a obtusa. Las flores son panículas racemosas de color verdoso, se desarrollan de yemas que aparecen al final o previa a la estación de crecimiento. ${ }^{45}$ La especie se distribuye en México desde Oaxaca (Istmo de Tehuantepec), sur de Tabasco, Campeche, Yucatán y Quintana Roo, formando parte importante en la estructura florística de la selva baja caducifolia. Es considerada como la especie más ampliamente distribuida en el centro y norte de la Península de Yucatán. ${ }^{46}$ Es de gran importancia en la industria apícola, la miel producida con esta especie es de alta estima. ${ }^{47}$

\section{Leucaena leucocephala}

Árbol o arbusto caducifolio o perennifolio, de 3 a 6 $\mathrm{m}$ (hasta $12 \mathrm{~m}$ ) de altura con un diámetro de hasta $25 \mathrm{~cm}$, copa redondeada, ligeramente abierta y rala, hojas alternas, bacinadas, de 9 a $25 \mathrm{~cm}$ de largo, verde grisáceas y glabras. Tronco usualmente torci- do y se bifurca a diferentes alturas. Ramas cilíndricas ascendentes. Desarrolla muchas ramas finas cuando crece aislado. Las flores se encuentran en cabezuelas, con 100 a 180 flores blancas, de 1.2 a $2.5 \mathrm{~cm}$ de diámetro, flor de 4.1 a $5.3 \mathrm{~mm}$ de largo, pétalos libres, el fruto se encuentra en vainas oblongas de color verde cuando están tiernas y cafés cuando maduras, conteniendo de 15 a 30 semillas. ${ }^{48}$ Es de amplia distribución en las regiones tropicales y subtropicales de México, en altitudes que van de 0 a $900 \mathrm{msnm}$. Prospera en ambientes adversos. Se adapta muy bien a las tierras bajas, crece desde sitios secos con $350 \mathrm{~mm} /$ año hasta húmedos con 2300 $\mathrm{mm} / \mathrm{año}$ y temperatura media anual de 22 a $30^{\circ} \mathrm{C}$. Es necesario un período seco de cuatro a seis meses. $^{49}$

\section{Lysiloma latisiliquum}

Es una árbol perteneciente a la familia Leguminosae, el árbol alcanza hasta $20 \mathrm{~m}$ de altura y $70 \mathrm{~cm}$ de diámetro normal, representativo de la selva mediana subperennifolia, particularmente abundante, frecuente, con alta regeneración natural en sitios con vegetación secundaria de 9 a 12 años de edad derivados de selvas medianas y bajas subperennifolias ${ }^{50}$, sobre suelos calizos y de bosque seco próximo a las costas, con buen drenaje y a elevaciones menores de 400 msnm. ${ }^{51}$ Un árbol de 15-20 m de altura, con diámetros de 60 a $70 \mathrm{~cm}$, de fuste recto, se ramifica desde un $50 \%$ de su altura total, copa redondeada, ramas ascendentes. La corteza gris pardusco, algunas lenticelas blancas y circulares. Tiene hojas compuestas, bipinnadas, de 11 a $20 \mathrm{~cm}$ incluyendo el pecíolo. Los frutos son vainas de 9 a $15 \mathrm{~cm}$ de largo, 2 a $4 \mathrm{~cm}$ de ancho, dehiscentes, en forma aplanada, agudas, de color café oscuro. ${ }^{51}$ 
Tabla 6 Contenido de algunos compuestos fitoquímicos en seis especies forrajeras consumidas por caprinos en Yucatán (g/ kg MS)

\begin{tabular}{|c|c|c|c|c|c|c|c|c|c|}
\hline Planta & Referencia & FT & TT & TC & $\mathbf{P C}$ & FDN & FDA & Lig & Toxicidad \\
\hline \multirow{3}{*}{ L. leucocephala. } & Alonso-Díaz et al. ${ }^{67}$ & 10.18 & 8.00 & 45.71 & & & & & \multirow{3}{*}{ Entre el 10 al 30\% de la dieta (Bustamante) ${ }^{68}$} \\
\hline & Alonso-Díaz et al. ${ }^{17}$ & & & 7.75 & & & & & \\
\hline & Ventura-Cordero, datos por publicar. & 4.43 & 1.54 & 16.18 & 23.67 & 35.27 & 23.67 & 11.14 & \\
\hline G. floribundum. & Ventura-Cordero, datos por publicar. & 7.95 & 0.75 & 33.84 & 9.65 & 48.7 & 35.36 & 16.66 & \multirow{2}{*}{$\begin{array}{c}\text { No disponible. } \\
\text { Subfamilia Mimosoideae se reporta en 1.5\% } \\
\text { del peso corporal en Rumiantes y perros } \\
\text { (Dep. Ecosystem science and management } \\
\text { Texas). }\end{array}$} \\
\hline M. bahamensis. & Ventura-Cordero, datos por publicar. & 6.15 & 1.06 & 32.01 & 14.55 & 44.31 & 20.02 & 9.23 & \\
\hline \multirow[t]{2}{*}{ A. cornígera. } & Rodríguez et al. ${ }^{69}$ & 31.1 & 15.0 & & & & & & $\begin{array}{c}\text { Se reporta para el género Acacia en rumian- } \\
\text { tes a partir de } 200 \mathrm{~g} \text { diarios por } 21 \text { días } \\
(\text { Odenyo et al. })^{70}\end{array}$ \\
\hline & Alonso-Díaz et al. ${ }^{17}$ & 28.45 & 24.41 & 46.91 & & & & & \multirow{3}{*}{ No disponible. } \\
\hline \multirow[t]{2}{*}{ L. latisiliquum. } & Martínez-Ortiz de Montellano et al. ${ }^{35}$ & 3,38 & 2,39 & 5,46 & 15.12 & & & & \\
\hline & Brunet et $a l . .^{71}$ & 59,38 & 33,41 & 9,02 & 14.9 & 49.10 & 25.10 & & \\
\hline A. gaumeri. & $\begin{array}{c}\text { Avello }^{72} \\
\text { Hernández-Orduño } \text { et al. }^{34} \\
\text { Alonso-Díaz et al. } .^{17}\end{array}$ & 86.77 & $\begin{array}{l}59.14 \\
53.91\end{array}$ & $\begin{array}{c}15.03 \\
12.2\end{array}$ & 21.47 & & & & $\begin{array}{l}\text { Se reporta para el género Acacia en rumian- } \\
\text { tes a partir de } 200 \mathrm{~g} \text { diarios por } 21 \text { días } \\
(\text { Odenyo } \text { et al. })^{70}\end{array}$ \\
\hline
\end{tabular}




\section{Mimosa bahamensis}

Un arbusto de la familia de las Fabáceas. Esta especie puede alcanzar hasta $10 \mathrm{~m}$ de altura. Presenta hojas bipinnadas, con raquis principal de 12 a $14 \mathrm{~cm}$ de longitud, 8 a 10 pares de hojuelas de 4 a $6 \mathrm{~cm}$ de largo y pecíolo acanalado de 0.5 a $2 \mathrm{~cm}$, cada hojuela con 22 a 32 pares de pinnas de 3 a $7 \mathrm{~mm}$ de largo y 7 a $17 \mathrm{~mm}$ entre par de pinnas. Inflorescencia en espiga con flores sésiles de corolas amarillas de 1.1 a $1.5 \mathrm{~mm}$ de largo, ligeramente más largas que el cáliz. Legumbre color caoba, usualmente erecta, rolliza, 5 a $9 \mathrm{~cm}$ de longitud por 1.3 a $1.8 \mathrm{~cm}$ de anchura, ápice puntiagudo, semillas color pardo obscuro. ${ }^{48}$ Poseen un par de espinas considerablemente infladas, ahuecadas hacia afuera, que están en pares, de más de $2 \mathrm{~cm}$ de largo, ubicadas en la base de cada hoja. Estas espinas generalmente son de tonalidad café, aunque algunas veces también se presentan de color marfil o amarillas. Los peciolos presentan nectarios y unos pequeños órganos nutritivos, denominados cuerpos de Belt que se localizan en el extremo de cada foliolo. ${ }^{26}$

Este tipo de acacia crece extraordinariamente rápido y son particularmente abundantes en áreas perturbadas, donde la competencia entre especies colonizadoras de rápido crecimiento es con frecuencia muy intensa. ${ }^{26}$ Tanto la flor como su fruto, se observa en el periodo de abril-agosto. ${ }^{52}$ En México se la observa en la Península de Yucatán, en la vertiente del Golfo desde Tabasco hasta Tamaulipas, mientras que en la del Pacifico es común en los estados de Chiapas, Oaxaca y Guerrero. ${ }^{52}$ Se le utiliza para fines medicinales, como combustible y como forraje ocasional. Es conocida por su relación simbiótica con las hormigas del género Pseudomyrmex ( $P$. ferruginea, $P$. belti, $P$. nigrosincta ${ }^{26,52}$
Arbusto que llega a medir $8 \mathrm{~m}$ de altura, hojas verdes muy pequeñas. Flores abundantes de color rosa o blanco, en forma de cabezas globosas. Se considera caliente. Crece a orilla de los caminos y en zonas de vegetación secundaria derivada de selvas bajas y medianas caducifolias, en selvas bajas subcaducifolias y selvas altas sub perennifolias. ${ }^{53}$ Tiene una amplia distribución en América, desde Brasil hasta México, se la encuentra en los estados de Campeche, Chiapas, Quintana Roo, Tabasco, Veracruz y Yucatán. ${ }^{52,53}$ Destaca su uso en el tratamiento de quemaduras y heridas superficiales de la piel y problemas gastrointestinales, en la construcción de cercas y postería, como combustible y para la recuperación de suelos y vegetación. ${ }^{52,53}$

\section{Acacia gaumeri}

Árbol de la familia de leguminosas que se distribuye en las selvas bajas, medianas y vegetación secundaria del sureste de México. ${ }^{54}$ Se le emplea para la elaboración de artículos menores de madera, y como forrajera. ${ }^{55}$ También se le conoce por su importante aportación a la industria apícola. Es considerada como uno de los mejores combustibles. ${ }^{54}$

Los parámetros algunos compuestos fenólicos se pueden observar en la tabla 6 .

\section{Conclusiones}

El uso masivo e inadecuado de compuestos antihelmínticos, han propiciado a nivel global la proliferación de poblaciones de NGI de rumiantes con resistencia. Lo anterior, aunado a una creciente concientización sobre la inocuidad alimentaria, así como también el impacto negativo de los fármacos 
sobre el medio ambiente, han despertado el interés por evaluar estrategias alternativas al uso de medicamentos sintéticos para el control de parásitos.

Entre estas opciones distintas al uso de fármacos, se encuentra el empleo de plantas ricas en compuestos bioactivos o metabolitos secundarios. Estos son compuestos fitoquímicos cuya función primaria está en la defensa de la planta contra herbívoros y patógenos. Desde hace tiempo se conoce que dichos compuestos bioactivos que contienen las leguminosas forrajeras tienen la capacidad de modificar diversos procesos biológicos de los nematodos gastrointestinales de rumiantes, principalmente la eclosión y desenvaine de larvas, así como el conteo fecal de huevecillos. Sin embargo, a pesar de que esta es una de las opciones más prometedoras, sobre todo en regiones tropicales, todavía existe un largo camino por recorrer, ya que el gran número de factores que influyen sobre la disponibilidad y concentración de MS, propicia una gran variabilidad en el efecto antihelmíntico in vivo e in vitro, sobre todo en esta última modalidad, por lo que es necesario continuar e incrementar las investigaciones que permitan conocer a fondo cuales compuestos generan mayor actividad antihelmíntica, así como también las especies de plantas que los contienen. Por otra parte, es importante señalar que hasta la fecha la opción más viable para proporcionar estos compuestos bioactivos a los rumiantes, es el pastoreo directo, por lo que también resulta necesario realizar evaluaciones a fin de poder determinar el manejo eficiente de los rumiantes. Por ultimo cabe resaltar que, si bien está ampliamente demostradas las propiedades antihelmínticas de una amplia gama de plantas, este no puede ser un método único en el control de NGI en rumiantes, por lo que deberá de ser empleado en combinación con otras estrategias.

\section{Conflictos de intereses}

La presente investigación no presenta conflictos de interés.

\section{Agradecimientos}

Al Campus de Ciencias Biológicas y Agropecuarias de la Universidad Autónoma de Yucatán.

Al Instituto Nacional de Investigaciones, Forestales, Agrícolas y Pecuarias

\section{Literatura citada}

1. Torres-Acosta JF, Molento M, Mendoza-de Gives P. Research and implementation of novel approaches for the control of nematode parasites in Latin America and the Caribbean: is there sufficient incentive for a greater extension effort? Vet Parasitol 2012; 186(1-2)132-42. https://doi. org/10.1016/j.vetpar.2011.11.053

2. Beltrão-Molento M, Silva-Fortes F, SantosPondelek DA, De Almeida-Borges F, De SouzaChagas AC, Torres-Acosta JF, et al. Challenges of nematode control in ruminants: Focus on Latin America. Vet Parasitol 2011; 180(1-2): 12632. https://doi.org/10.1016/j.vetpar.2011.05.033

3. Hoste H, Jackson F, Athanasiadou S, Thamsborg MS, Hoskin OS. The effects of tannin-rich plants on parasitic nematodes in ruminants. Trends Parasitol 2006; 22(6):253-61. https://doi.org/10. 1016/j.pt.2006.04.004

4. Mendoza de Gives P, Lopez-Arellano ME, Liebano-Hernandez E, Aguilar-Marcelino L. 2012. Cap. 6 Plant Extracts: a Potential Tool for Controlling Animal Parasitic Nematodes en The Biosphere, editado por Natarajan Ishwaran, 314 pags. https://mts.intechopen.com/books/the-bios 
phere/plant-extracts-a-potential-tool-for-contro lling-animal-parasitic-nematodes.

5. Hammond JA, Fielding D, Bishop SC. Prospects for plant anthelmintics in tropical veterinary medicine. Vet Res Commun 1997;21(3):213-28.

6. Jackson F, Coop RL. The development of anthelmintic resistance in sheep nematodes. Parasitology 2000;120(7):95:107.

7. Burke JM, Miller JE, Olcott DD, Olcott BM, Terrill TH. Effect of copper oxide wire particles dosage and feed supplement level on Haemonchus contortus infection in lambs. Vet Parasitol 2004;123(3-4):235-43.

8. Torres-Vásquez P, Prada-Sanmiguel VG, Márquez-Lara D. Resistencia antihelmíntica en nematodos gastrointestinales del bovino. Rev Med Vet 2007;13:59-76.

9. Sandoval-Castro CA, Torres-Acosta JFJ, Hoste H, Salem AZM, Chan-Pérez JI. Using plant bioactive materials to control gastrointestinal tract helminths in livestock. Anim Feed Sci Technol 2012;176(1-4):192-201.

https://doi.org/10.1016/j.anifeedsci.2012.07.023

10.Githiori JB, Athanasiadou S, Thamsborg MS. Use of plants in novel approaches for control of gastrointestinal helminthes in livestock with emphasis on small ruminants. Vet Parasitol 2006;139(4):308-20. https://doi.org/10.1016/j. vetpar.2006.04.021

11.Hernández-Villegas MM, Borges-Argáez R, Rodríguez-Vivas RI, Torres-Acosta JF, Méndez-González M, Cáceres-Farfán M. Ovicidal and larvicidal activity of the crude extracts from Phytolacca icosandra against Haemonchus contortus. Vet Parasitol 2011;179(1-3): 100-6. https://doi.org/10.1016/j.vetpar.2011.02.019

12.Vargas-Magaña JJ, Aguilar-Caballero AJ, Torres-Acosta JF, Sandoval-Castro CA, Hoste H,
Capetillo-Leal CM. Tropical tannin-rich fodder intake modifies saliva-binding capacity in growing sheep. Animal 2013;7(12):1921-4. https://doi.org/10.1017/S1751731113001651

13. Salem AZM, Lopez S, Robinson PH. Plant bioactive compounds in ruminant agriculture - Impacts and opportunities. Anim Feed Sci Technol 2012; 176(1-2):1-4. https://doi.org/10.1016/j.ani feedsci.2012.07.011

14.Ramírez-Restrepo CA, Barry TN. Alternative temperate forages containing secondary compounds for improving sustainable productivity in grazing ruminants. Anim Feed Sci Tecnol 2005;120 (3-4): 179-201. http://dx.doi.org/10. 1016/j.anifeedsci.2005.01.015

15.Calderón-Quintal JA, Torres-Acosta JFJ, Sandoval-Castro CA, Alonso MA, Hoste H, AguilarCaballero A. Adaptation of Haemonchus contortus to condensed tannins: can it be possible? Arch Med Vet 2010;42(3):165-71.

16. Max RA, Wakelin D, Dawson JM, Kimambo AE, Kassuku AA, Mtenga LA, et al. Effect of quebracho tannin on faecal egg counts and worm burdens of temperate sheep with challenge nematode infections. J Agric Sci 2005;143(6):51927. https://doi.org/10.1017/S0021859605005691

17.Alonso-Diaz MA, Torres-Acosta JFJ, SandovalCastro CA, Hoste H. Comparing the sensitivity of two in vitro assays to evaluate the anthelmintic activity of tropical tannin rich plant extracts against Haemonchus contortus. Vet Parasitol 2011;181(2-4):360-4. https://doi:10.1016/j.vet par.2011.03.052

18.González-Pech PG, Torres-Acosta JFJ, Sandoval-Castro CA, Tun-Garrido J. Feeding behavior of sheep and goats in a deciduous tropical forest during the dry season: The same menu consumed differently. Small Rumin Res 2015;133:128-34. 
https://doi.org/10.1016/j.smallrumres.2015.08.02 $\underline{0}$

19.Ávalos-Garcia A, Pérez-Urria Carril E. Metabolismo secundario de plantas. Reduca (Biología). Serie Fisiología Vegetal 2009; 2(3):119-45.

20.Lincoln T, Zeiger E. Fisiología Vegetal Vol. 1. Edit. Universitat Jaume. Tercera Edición; España. 2006. p. 584.

21.Isaza JH. Taninos o polifenoles vegetales. Sci Tech 2007; 13(33):13-8.

22.Kroymann J. Natural diversity and adaptation in plant secondary metabolism. Curr Opin Plant Biol 2011;14(3):246-51. https://doi.org/10. 1016/j.pbi.2011.03.021

23.Sepúlveda-Jimenéz G, Porta-Ducoing H, RochaSosa M. La Participación de los metabolitos secundarios en la defensa de las plantas. Rev Mex Fitopatol 2003;21(3):355-63.

24.Valares-Masa C. Variación del metabolismo secundario en plantas debida al genotipo y al ambiente. [Tesis doctoral]. Universidad de Extremadura. España; 2011. p. 216.

25.Zaynab M, Fatima M, Abbas S, Sharif Y, Umair M, Zafar MH, Bahadar K. Role of secondary metabolites in plant defense against pathogens. Microb Pathog 2018; 124: 198-202. https://doi. org/10.1016/j.micpath.2018.08.034

26. Raven PH, Ever RF, Eirchhorn SE. Biología de las plantas II. Editorial Reverte. España; 1992. p. 773.

27.Ramírez-Lozano RG. Alimentación del venado cola blanca: Biología y ecología nutricional. Editorial Palibrio. Primera Edición; 2012. p.356.

28.Villalba JJ, Provenza FD, Manteca X. Links between ruminants' food preference and their welfare. Animal 2010; 4(7):1240-7. https://doi. org/10.1017/S1751731110000467

29. Costa CTC, Bevilaqua CML, CamurçaVasconcelos ALF, Maciel MV, Morais SM, Cas- tro CMS, et al. In vitro ovicidal and larvicidal activity of Azadirachta indica extracts on Haemonchus contortus. Small Rumin Res 2008;74(1-3):284-7. $\quad$ https://doi.org/10.1016/j. smallrumres.2007.09.003

30.Lisonbee LD, Villalba JJ, Provenza FD, Hall JO. Tannins and self-medication: Implications for sustainable parasite control in herbivores. Behav Processes 2009;82(2):184-9. https://doi.org/10. 1016/j.beproc.2009.06.009

31.Hoste H, Martínez-Ortiz-De-Montellano C, Manolaraki F, Brunet S, Ojeda-Robertos N, Fourquaux I, et al. Direct and indirect effects of bioactive tannin-rich tropical and temperate legumes against nematode infections. Vet Parasitol 2012;186(1-2):18-27. https://doi.org/10.1016/j. vetpar.2011.11.042

32.von Son-de Fermex E, Alonso-Díaz MA, Vallesde la Mora B, Capetillo Leal CM. In vitro anthelmintic activity of five tropical legumes on the exsheathment and motility of Haemonchus contortus infective larvae. Exp Parasitol 2012;131(4):413-8. $\quad$ https://doi.org/10.1016/j. exppara.2012.05.010

33. Azmir J, Zaidul ISM, Rahman MM, Sharif KM, Mohamed A, Sahena F, et al. Techniques for extraction of bioactive compounds from plant materials: A review. J Food Eng 2013;117(4) 426:36. https://dx.doi.org/10.1016/j.jfoodeng. 2013.01.014

34.Hernández-Orduño G, Torres-Acosta JFJ, Sandoval-Castro CA, Aguilar-Caballero AJ, ReyesRamirez RR, Hoste $\mathrm{H}$, et al. In vitro anthelmintic effect of Acacia gaumeri, Havardia albicans and Quebracho tannin extracts on a Mexican strain of Haemonchus contortus L3 larvae. Trop Subtrop Agroecosyst 2008;8(2):191-7.

35.Martínez-Ortiz-de-Montellano C, Vargas-Maga ña JJ, Canul-Ku HL, Miranda-Soberanis R, Ca- 
petillo-Leal C, Sandoval-Castro CA, et al. Effect of a tropical tannin-rich plant Lysiloma latisiliquum on adult populations of Haemonchus contortus in sheep. Vet Parasitol 2010;172(34):283-90. https://doi.org/10.1016/j.vetpar.2010. $\underline{04.040}$

36. Galicia-Aguilar HH, Rodríguez-González LA, Capetillo-Leal CM, Cámara-Sarmiento IR, Aguilar-Caballero AJ, Sandoval-Castro CA, et al. Effects of Havardia albicans supplementation on feed consumption and dry matter digestibility of sheep and the biology of Haemonchus contortus. Anim Feed Sci Technol 2012;176(1-4):178-84. https://doi.org/10.1016/j.anifeedsci.2012.07.021

37.Méndez-Ortiz FA, Sandoval-Castro CA, TorresAcosta JFJ. Short term consumption of Havardia albicans tannin rich fodder by sheep: Effects on feed intake, diet digestibility and excretion of Haemonchus contortus eggs. Anim Feed Sci Technol 2012;176(1-4):185-91.

38. Ventura-Cordero J, Pech-Cervantes A, Sandoval-Castro CA, Torres-Acosta JFJ, GonzalezPech PG, Sarmiento-Franco LA. Relación herbívoro-tanino: adaptación de ovinos y caprinos a la vegetación rica en taninos de la Península de Yucatán. Bioagrociencias 2013; 6(1):19-25.

39. Taylor MA, Hunt KR, Goodyear KL. Anthelmintic resistance detection methods. Vet Parasitol 2002;103(3)183-94.

40.Coles GC, Jackson F, Pomroy WE, Prichard RK, von Samson-Himmelstjerna G, Silvestre A, et al. The detection of anthelmintic resistance in nematodes of veterinary importance. Vet Parasitol 2006;136(3-4):167-85. https://dx.doi.org/10. 1016/j.vetpar.2005.11.019

41.Sosa-Rubio EE, Cabrera-Torres E, PérezRodríguez D. El uso de la vegetación secundaria (acahuales) para la alimentación de bovinos y ovinos en Quintana Roo. Folleto Técnico. INIFAP-CIRSE-CE Chetumal; 2006. p. 22.

42. Bailey WD, Gross JE, Laca EA, Rittenhouse LR, Coughenour BM, Swift DM, et al. Mechanisms that result in large herbivore grazing distribution patterns. J Range Manage 1996; 49:386-400.

43. Cotler-Avalos H. Las cuencas hidrográficas de México. Diagnóstico y priorización. Instituto Nacional de Ecología; 2010. p. 232.

44.González-Pech PG, Torres-Acosta JFJ, Sandoval-Castro CA. Adapting bite coding grid for small ruminants browsing a deciduous tropical forest. Trop Subtrop Agroecosyt 2014;17(1): 6370.

45.Interián-Ku VM, Valdez-Hernández JI, GarcíaMoya E, Romero-Manzanares A, Borja-de-laRosa MA, Vaquera-Huerta H. Arquitectura y morfometría de dos especies arbóreas en una selva baja caducifolia del sur de Yucatán, México. Bol Soc Bot Méx 2009; (85): 17-29.

46.Balam-Narváez R. Una planta común con varias funciones. Aunque usted no lo crea!!. 2009. Disponible en http://www.cicy.mx/Documentos/CI CY/Desde_Herbario/2009/Septiembre/Gymnopo dium\%20Septiembre\%2010\%202009.pdf. (Consultado el 18 de Mayo de 2013).

47.Moguel-Ordóñez YB, Echazarreta-González C, Mora-Escobedo R. Calidad fisicoquímica de la miel de abeja Apis mellifera producida en el estado de Yucatán durante diferentes etapas del proceso de producción y tipos de floración. Téc Pecu Méx 2005; 43:323-34.

48.Comisión Nacional de Biodiversidad [sede Web]. México, 2013 [consultado septiembre 2018]. Disponible en: http://www.conabio. gob.mx/malezasdemexico/mimosaceae/leucaenaleucocephala/fichas/ficha.htm. 
49.Gispert-Cruells M, Rodríguez-González H, González-Esquinca AR. Los diversos y floridos arboles de los parques de Tuxtla Gutiérrez, Chiapas. Universidad Nacional Autonoma, Mexico. 2002. p. 102.

50.López-Torres JL, Tamarit-Urias JC. Crecimiento e incremento en diámetro de Lysiloma latisiliquum (L.) Benth en bosques secundarios en Escárcega, Campeche, México. Rev Chapingo Ser Cienc For Ambient 2005;11(2): 117-23.

51.Cordero J, Boshier DH. Árboles de Centroamérica: un manual para extensionistas. CATIE/FPR, Turrialba, Costa Rica; 2003.

52.Centro de Investigación Científica de Yucatán [sede Web]. Mexico, 2013 [consultado 28 de septiembre 2018]. Disponible en: http://www. cicy.mx/sitios/flora\%20digital/ficha_virtual.php? especie $=1491$.

53.Instituto Nacional de Ecología. México, 2013. [consultado 18 de mayo de 2013]. Disponible en: http://www1.inecol.edu.mx/publicaciones/resum eness/flover/147-martinez_bernal.pdf.

54.Arellano-Rodríguez JA. Etnoflora yucatanense fascículo 20. Nomenclatura, forma de vida, uso, manejo y distribución de las especies vegetales de la península de Yucatán. Universidad Autónoma de Yucatán; México. 2003. p. 819.

55.Flores JS, Bautista F. El Conocimiento de los mayas yucatecos en el manejo del bosque tropical estacional: las plantas forrajeras. Rev Méx Biodivers 2012;83(2):503-18.

56.Kamaraj C, Rahuman AA. Efficacy of anthelmintic properties of medicinal plant extracts against Haemonchus contortus. Res Vet Sci 2011;91(3):400-4. https://doi.org/10.1016/j.rvsc. $\underline{2010.09 .018}$

57. Marie-Magdeleine C, Udino L, Philbert L, Bocage $\mathrm{B}$, Archimede $\mathrm{H}$. In vitro effects of Cassava (Manihot esculenta) leaf extracts on four devel- opment stages of Haemonchus contortus. Vet Parasitol 2010;173(1-2):85-92. https://doi.org/ 10.1016/j.vetpar.2010.06.017

58.Silva MJD, Carvalho AJS, Rocha CQ, Vilegas W, Silva MA, Gouvêa CMCP. Ethanolic extract of Mimosa caesalpiniifolia leaves: Chemical characterization and cytotoxic effect on human breast cancer MCF-7 cell line. S Afr J Bot 2014;93:64-9. https://doi.org/10.1016/J.SAJB. 2014.03.011

59. Monteiro MV, Bevilaqua CM, Morais SM, Machado LK, Camurça-Vasconcelos AL, Campello CC, et al. Anthelmintic activity of Jatropha curcas L. seeds on Haemonchus contortus. Vet Parasitol 2011;182(2-4):259-63. https://doi.org/10. 1016/j.vetpar.2011.04.010

60.Chen CY, Wang YD. Secondary metabolites from Leucaena leucocephala. Chem Nat Compd 2011;47(1):145-6. https://dx.doi.org:10.1007/s 10600-011-9862-Z

61. Chew YL, Chan EW, Tan PL, Lim YY, Stanslas J, Goh JK. Assessment of phytochemical content, polyphenolic composition, antioxidant and antibacterial activities of Leguminosae medicinal plants in Peninsular Malaysia. BMC Complement Altern Med 2011;11: 12. https://dx.doi. org/10.1186/1472-6882-11-12

62. Alhaji UI, Samuel NU, Aminu M, Chidi AV, Umar ZU, Umar UA, et al. In vitro antitrypanosomal activity, antioxidant property and phytochemical constituents of aqueous extracts of nine Nigerian medicinal plants. Asian Pac J Trop Dis 2014; 4(5):348-55. https://doi.org/10.1016/ S2222-1808(14)60586-7

63.Covarrubias-Cárdenas AG, Torres-Acosta JFJ, Sandoval-Castro CA, Hoste H. 2013. In vitro anthelmintic effect of Acacia pennatula and Coffea arabica extracts on Haemonchus contortus sensitive to tannins. En memorias de la 7th Novel 
Approaches Meeting with a session of the CAPARA COST ACTION "Goat parasite interaction: from knowledge to control". Toulouse, Francia.

64. Ortiz-Ocampo GI, Torres-Acosta JFJ, SandovalCastro CA, Hoste H. 2013. In vitro anthelmintic activity of Acacia pennatula and Coffea arabica against Haemonchus contortus showing low susceptibility to tannins from tropical forages. En memorias de la 7th Novel Approaches Meeting with a session of the CAPARA 38 COST ACTION "Goat parasite interaction: from knowledge to control". Toulouse, Francia.

65.Martínez-Ortiz-de-Montellano C, Arroyo-López C, Fourquaux I, Torres-Acosta JF, SandovalCastro CA, Hoste H. Scanning electron microscopy of Haemonchus contortus exposed to tannin-rich plants under in vivo and in vitro conditions. Exp Parasitol 2013;133(3): 281-6. https:// doi.org/10.1016/j.exppara.2012.11.024

66.Eguale T, Tilahun G, Debella A, Feleke A, Makonnen E. In vitro and in vivo anthelmintic activity of crude extracts of Coriandrum sativum against Haemonchus contortus. J Ethnopharmacol 2007; 110(3): 428-33. https://dx.doi.org/10. 1016/j.jep.2006.10.003

67.Alonso-Diaz MA, Torres-Acosta JF, SandovalCastro CA, Aguilar-Caballero AJ, Hoste H. In vitro larval migration and kinetics of exsheathment of Haemonchus contortus larvae exposed to four tropical tanniniferous plant extracts. Vet Parasitol 2008; 153(3-4):313-9. https://www.doi/ 10.1016/j.vetpar.2008.01.042
68. Bustamante GJJ. Producción de leche en pastoreo suplementado con leucaena en áreas compactas. Folleto Científico No. 3. INIFAPCIRPAC; 2000. p. 31.

69.Rodríguez R, Britos A, Rodríguez-Romero N, Fondevilla M. Effect of plant extracts from several tanniferous browse legumes on in vitro microbial fermentation of the tropical grass Pennisetum purpureum. Anim Feed Sci Technol 2011 168:188-95. https://doi.org/10.1016/j. anifeedsci.2011.04.095

70. Odenyo AA, Osuji PO, Karanfil O, Adinew K. Microbiological evaluation of Acacia angustissima as a protein supplement for sheep. Anim Feed Sci Technol 1997; 65: 99-112. https://doi. org/10.1016/S0377-8401(96)01087-5

71.Brunet S. de Montellano CM, Torres-Acosta JF, Sandoval-Castro CA, Aguilar-Caballero AJ, Capetillo-Leal C, Hoste H. Effect of the consumption of Lysiloma latisiliquum on the larval establishment of gastrointestinal nematodes in goats. Vet Parasitol 2008;157(1-2):81-8. https://dx.doi. org/10.1016/j.vetpar.2008.07.013

72. Avello LM, Cisternas HI. Fitoterapia, sus orígenes, características y situación en Chile. Rev Med Chile 2010; 138:1288-93. https://dx.doi. org/10.4067/S0034-98872010001100014 